\title{
Valorando la RSE por los stakeholders-internos: caso sector de la construcción en Bucaramanga
}

CSR assessment through stakeholders-internal: the case of the construction sector in Bucaramanga 


\title{
Valorando la RSE por los stakeholders-internos: caso sector de la construcción en Bucaramanga'
}

\author{
CSR assessment through stakeholders-internal: the case of the \\ construction sector in Bucaramanga
}

\author{
Andrés Mauricio García Gómez², Alba Guzmán Duque³, \\ Javier Mendoza Paredes ${ }^{4}$
}

Unidades Tecnológicas de Santander, Bucaramanga, Colombia

Artículo recibido en agosto de 2016; artículo aceptado en octubre de 2016

\begin{abstract}
Citación del artículo: García. A., Guzmán. A., \& Mendoza, J. (2017). Valoración de la RSE a través de los stakeholders-
\end{abstract}
internos: el caso del sector de la construcción en Bucaramanga. I+D Revista de Investigaciones, 9(1), 107 - 115

\begin{abstract}
Resumen
La responsabilidad social empresarial (RSE) orienta a las organizaciones a interactuar con sus stakeholders, creando valor en el mercado a través de la satisfacción de sus clientes externos -el gobierno, la sociedad, los proveedorespero desconociendo el valor que el cliente interno aporta a la organización. Ambos grupos generan impactos para las empresas en la toma de decisiones, aunque los stakeholders internos-accionistas, socios aliados y trabajadores- son subvalorados, pese a que son un componente importante para la sostenibilidad organizacional puesto que favorecen su imagen corporativa. En este artículo se presentan los resultados de una investigación realizada en sesenta empresas del sector de la construcción en Bucaramanga en el año 2013, donde se evidencia que los objetivos de las empresas se orientan a satisfacer al cliente externo para mantener su posición competitiva en el mercado, ignorando el impacto que producen sus clientes internos para la mejora de sus procesos. De hecho, en las empresas se generan valores positivos para el cumplimiento de las necesidades de sus accionistas, sin intervenir en los trabajadores como elemento fundamental del proceso para generar valor al cliente externo. Tras ese análisis se realizan sugerencias a los empresarios para incluir dentro de sus organizaciones buenas prácticas que fomenten la valoración de los stakeholders -internos, como encargados de diseminar y fortalecer la imagen de la compañía.
\end{abstract}

Palabras clave: Stakeholders-internos, sostenibilidad, responsabilidad social empresarial, valoración de las empresas.

1. Artículo de investigación con enfoque cualitativo, resultado del proyecto de investigación Metodología de Diagnóstico, formación, asistencia técnica y acompañamiento que posibilite la formulación e implementación de un modelo de gestión basado en responsabilidad social y empresarial para las mipymes del sector de la construcción en la ciudad de Bucaramanga (culminado), perteneciente al área de Ciencias socioeconómicas, responsabilidad social empresarial. Desarrollado en el grupo de investigación Gicse y financiado por las Unidades Tecnológicas de Santander de la ciudad de Bucaramanga (Colombia). Dirección: Calle de los Estudiantes n. ${ }^{\circ}$ 9-82, Bucaramanga, Colombia, PBX: 6917700. Fecha de inicio: 2013, fecha de terminación: 2014.

2. Administrador de Empresas, Universidad Cooperativa de Colombia. Magister en Dirección Estratégica, Universidad Internacional Iberoamericana. Docente- investigador del grupo Gicse. Unidades Tecnológicas de Santander de la ciudad de Bucaramanga (Colombia): Dirección: Calle de los Estudiantes 9-82, PBX:6917700. Correo institucional: agarcia@correo.uts.edu.co

3. Administradora de Empresas, Universidad Nacional de Colombia. PhD. en Gestión de las TIC, Universidad Politécnica de Valencia. Docenteinvestigadora y líder del grupo Gicse. Unidades Tecnológicas de Santander de la ciudad de Bucaramanga (Colombia): Dirección: Calle de los Estudiantes n. 9-82, PBX: 6917700. Correo institucional: aguzman@correo.uts.edu.co

4. Administrador de Empresas, Universidad Autónoma de Bucaramanga, Magister en Administración, Universidad Autónoma de Bucaramanga -Instituto Tecnológico de Monterrey. Docente- investigador del grupo Gicse. Unidades Tecnológicas de Santander de la ciudad de Bucaramanga (Colombia): Dirección: Calle de los Estudiantes n. 9-82, PBX: 6917700. Correo electrónico institucional: jmendoza@correo.uts.edu.co 


\begin{abstract}
The Corporate Social Responsibility (CSR) guides organizations to interact with their stakeholders, seeking to satisfy the external in order to create value in the market, considering government, society, and providers, among others. However, the internal customer is neglected, ignoring the value that brings to the organization. Both groups generate impacts for companies in decision-making, although internal stake holders [shareholders, alliance partners and workers] are an important component for organizational sustainability, since they favor the corporate image. This article presents the results of a research conducted on 60 companies within the construction sector in Bucaramanga in 2013, where there is evidence that the business objectives are oriented to satisfy the external customer in order to maintain their competitive position in the market, ignoring the impact upon their internal stakeholders. In addition, positive values are generated to meet the needs of its shareholders, without interfering with its employees, as a fundamental element of the process of generating value to the external stakeholders. After this analysis suggestions are made to entrepreneurs to include the best practices within their organizations to promote the valorization of internal customers, such as disseminating and strengthening the image of the company.
\end{abstract}

Keywords: Stakeholders, sustainability, corporate social responsibility, valuation of companies.

La responsabilidad social empresarial (RSE) es un campo de acción dentro y fuera de la organización, direccionado a partir de dos enfoques: holístico-filantrópico y estratégico. En la mirada holística-filantrópica la empresa orienta su quehacer hacia actividades que generan un beneficio propio en el sector externo, planteando acciones que conduzcan a la fidelización del cliente para lograr la sostenibilidad empresarial en todo su campo de acción. De otro lado, el enfoque estratégico determina a la RSE como la estrategia del negocio, proporcionando mayor responsabilidad del sector interno, en diferentes funciones y actividades que han sido formuladas, creadas y puestas en marcha para la satisfacción del cliente interno (Caamaño, 2012). Lo anterior permite identificar dos grupos de interés o stakeholders: el interno y el externo.

En este artículo se analiza el aporte que hacen los stakeholders-internos a las empresas del sector de la construcción de la ciudad de Bucaramanga, mostrando que se requiere prestar una mayor atención, pues son los responsables de los procesos que permiten a las organizaciones ser competitivas en el sector; finalmente, se presenta a manera de propuesta, la valoración que debería realizar la empresa a través de la RSE para cumplir con sus objetivos.

\section{Aproximación teórica}

\section{Conceptualización de la RSE}

La responsabilidad social empresarial (RSE) se enfrenta a una variedad conceptual para favorecer el presente y el futuro de las empresas enfocadas en diferentes aspectos: la filantropía y el altruismo, las esferas estratégicas de desarrollo empresarial, una mayor conciencia ética y la comprensión del significado de la sostenibilidad. Este hecho implica que se presenten diversas definiciones de la RSE (Aguilera \& Puerto, 2012; McWilliams, Siegel \& Wright, 2006). Por ejemplo, Perlines (2015) indica que la RSE es un esquema en donde las organizaciones tienen compromisos para favorecer colectivos sociales, que están por encima de las empresas, y que a la vez cumplen con las normativas de ley, considerando las diferentes tendencias mundiales que incluye la RSE en la cotidianidad. Para el Instituto Ethos de Empresa y Responsabilidad Social, (2011) y Ríos (2013) es una relación entre lo ético de la empresa y todo estamento público que le rodea, determinando metas empresariales unidas al desarrollo sostenible de una sociedad, el cuidado de los recursos ambientales y culturales en pro del desarrollo de las generaciones futuras, y la aceptación de la diversidad para buscar la reducción de las desigualdades sociales que impliquen también a las políticas gubernamentales.

De otro lado, una mirada de los autores hacia el interior de la organización hace otra aproximación relacionada con la definición de RSE. Suárez (2011) sostiene que la RSE se enfoca en la estrategia de gestión en el campo de la industria, de los gobiernos, de los sindicatos, de los trabajadores y de las organizaciones no gubernamentales, que se relacionan en los siguientes procesos: ambientales, sociales y económicos; y que conllevan a las organizaciones a ser responsables con sus comunidades, sus proveedores y los stakeholdersexternos. Por su parte, Accinelli y De la Fuente (2013) indican que la RSE es una fórmula donde interactúan los accionistas y los directivos (shareholder-internos) y su relación con los grupos de interés externos (stakeholdersexternos). Kowszyk, Covarrubias y García (2011) afirman que es el compromiso que asume una empresa para contribuir al desarrollo económico sostenible por medio de la colaboración con sus empleados, sus familiares, 
la comunidad local y la sociedad, con el objetivo de mejorar la calidad de vida de los ciudadanos. Jiménez, Alcaraz y Leco (2015) afirman que precisamente esos grupos internos favorecen la aplicación de las políticas de RSE siempre y cuando reciban por parte de la empresa capacitación como mecanismo para el desarrollo social.

En este sentido, y considerando los dos enfoques de la RSE, Bestratén y Pujol (2004) clasifican a la RSE en tres categorías: primaria, hace referencia a las actividades propias de las empresas, comercializar productos de calidad a un precio aceptable, creando plusvalía en la organización y la sociedad; secundaria, requiere del esfuerzo de la empresa porque va más allá de lo obligatorio puesto que divide estas responsabilidades en aquellas que se relacionan con los trabajadores y con las comunidades; $y$, terciaria, representa las acciones que no están incluidas en las actividades específicas de la empresas.

En la práctica, muchas de las responsabilidades terciarias son desarrolladas antes de asumir las primarias o las secundarias, porque se relacionan directamente con lo social y causan un mayor impacto en el ámbito externo (por ejemplo, los medios de comunicación). De hecho, García et al. (2016) indican que las pequeñas y medianas empresas del sector de la construcción están implementando procesos basados en RSE.

Por otra parte, entre las responsabilidades más comunes relacionadas con la comunidad Solano (2005) menciona que se orienta hacia:

-Proporcionar beneficios en alimentación fuera del hogar (ejemplo, comedores populares).

-Facilitar ayudas para vivienda (urbanizaciones, agua, alcantarillado, entre otras).

-Aportar herramientas para el desarrollo de actividades culturales y recreativas (cines, parques, entre otras).

-Incentivar actividades que beneficien a la comunidad en su contexto.

- Colaborar con centros de formación, como escuelas, universidades, entre otros.

-Promover la construcción en infraestructura.

-Patrocinar artistas, escritores, o científicos para el desarrollo de su obra.

De otro lado, es importante presentar la relación de los aspectos misionales de la empresa con la responsabilidad social empresarial. Correa, Flynn y Amit (2004) afirman que la relación con la misión de la empresa y la ética empresarial se enfoca en los elementos públicos en el cumplimiento de las metas organizacionales, buscando que sean compatibles con el desarrollo de la sociedad, en unión respetable con la diversidad para lograr una reducción de las desigualdades sociales. Guédez (2008) afirma que la RSE debe ser transparente en todas sus actividades y acciones, en especial en la función financiera, evidenciando libertad y disponibilidad de la información para quien la necesite; de esta manera, se alinean las estrategias organizacionales con la misión, la visión, los valores empresariales y la cultura empresarial.

Es importante indicar que existen diferentes elementos para identificar los grupos de interés en una organización, y que tienen presente a los stakeholders - internos y externos-, como son: por cercanía, se relacionan de manera cercana con la organización; por influencia, generan dominio en el desarrollo de la actividad de la empresa; por responsabilidad, cumplen obligaciones legales; $y$, por dependencia, se relacionan con el objeto social de la organización. Esta información le proporciona a la empresa datos relevantes para tomar decisiones, considerando que deben desarrollar agrupaciones de los stakeholders por características que muestren similitud y cercanía en las actividades para integrarlos desde un mismo orden dentro de la empresa y crear grupos multidisciplinarios que entreguen soluciones con una perspectiva global. Citando textualmente a Revilla y Fernández (2011, pp. 71-76) sobre los stakeholders "... de esta forma se identifica a cada grupo dependiendo de las actividades a desarrollar, como agente activo en la empresa o como agente externo que se integra en todo un proceso de la Responsabilidad Social Empresarial".

\section{Grupos de interés implicados en la RSE}

Las diferentes actividades y funciones que realizan las empresas se direccionan hacia una gestión propia de la organización para el cumplimiento estratégico y su relación con los grupos de interés en el desarrollo de la RSE. Revilla y Fernández (2011) los identifican en un contexto histórico, relacionándolos con los agentes económicos más cercanos y directos: inversionistas, clientes, proveedores; e identifican que la relación entre ellos ha evolucionado en diferentes escenarios de la vida económica y productiva de las organizaciones, siendo incluyentes en el proceso que favorece la sostenibilidad de las empresas.

Los grupos de interés internos se identifican activamente y de forma legítima en los elementos esenciales de la empresa influyendo en la toma de decisiones, Navarro (2012), incluyendo el interior y el exterior de la organización. Dentro de los mecanismos que se crean en el ámbito internacional, se identifican variables que facilitan la medición de la calidad de la relación entre la empresa y los stakeholders-internos de la organización 
como los colaboradores, la familia y los sindicatos, así como los stakeholders-externos, por ejemplo la Guía GRI, el Libro Verde de la Unión Europea, el Balance Social de la OIT, la Guía Confecámaras-BID y las Normas SA:8000 e ISO:26000- (Ulloque \& Berrio, 2014). En este sentido, se encuentran dos dimensiones de la RSE: la interna y la externa.

\section{Dimensión externa de la RSE}

Según Navarro (2012) los stakeholders-externos son grupos que no están en función directa de la empresa y se identifican como los consumidores, el gobierno, la competencia, los grupos de presión, entre otros. Para Aguilar, Becerra y Doria (2012) citando las investigaciones de Bateman y Snell realizadas en el año 2005 , los proveedores son quienes muestran un poder en relación con el trato, el régimen y las actividades comerciales sobre las empresas, esto implica el manejo y la dirección de situaciones en un beneficio propio generando ganancias para ambos. Por otro lado, el gobierno y la comunidad, son miembros vivos a su alrededor que promueven diferentes actividades para el seguimiento de una relación activa en beneficio de todos los miembros de una organización. Silos y Galiano (2011) consideran que con la RSE se integran la empresa y los consumidores dentro de un diálogo entre la organización y los stakeholders-externos dentro de la cadena productiva de la empresa buscando unir esfuerzos para su bienestar.

\section{Dimensión interna de la RSE}

El crecimiento y desarrollo de los grupos de interés internos que intervienen en el proceso de consolidación organizacional ha evolucionado, favoreciendo su unión con el contexto socio-económico en diferentes valores corporativos como la transparencia, la comunicación y el diálogo; al integrarse estos valores dentro de la estrategia corporativa se generan ventajas competitivas que aportan al mejoramiento del clima organizacional logrando un aprendizaje e innovación activa dentro de la empresa (Revilla \& Fernández, 2011), en el cual los grupos internos en la organización se relacionan con los empleados, los accionistas y los directivos (Accinelli \& De la Fuente, 2013). En este sentido, Jiménez, Alcaraz y Leco (2015) afirman que los stakeholders-internos favorecen la aplicación de las políticas estructurales de la empresa en el exterior. La Unión Europea (2001) en su publicación del Libro Verde de la Responsabilidad Social Empresarial manifiesta que la RSE no puede gestarse sin el cumplimiento y desarrollo del talento humano, que es un factor indispensable para alcanzar los objetivos organizacionales enfocados en el cumplimiento de su RSE. (Yfarraguirre, 2014)

\section{Valoración de la RSE}

La valoración de las empresas que se han integrado hacia los modelos de responsabilidad social empresarial y se direccionan hacia la sostenibilidad empresarial (García, et al., 2016) buscando la unión sistemática en sus procesos administrativos para ser adaptadas en modelos propios de RSE, considerando también la variable ambiental (Artalejo, 2010; Meza, 2011). Desde este punto procedimental, es necesaria la inclusión de herramientas para la medición de RSE que permitan determinar la influencia de estos factores en el comportamiento organizacional. En la publicación del Libro Verde de la RSE se indica textualmente que la "inversión interna dentro de una RSE se valora por medio de inversión en el talento humano, incluyendo su objetivos personales, su entorno familiar, profesional, sus necesidades básicas, y seguir avanzando en esa escala valorativa hasta cumplirlas en un ciento por ciento". (Unión Europea, 2001, p. 8)

De otro lado, para valorar la RSE existen herramientas que son aplicadas en el contexto de la organización. Meza (2011) indica que para que la medición sea adecuada, es necesario determinar un enfoque multi-stakeholders, que permita la alineación de las causas sociales siendo flexibles para ser restaurados en diferentes mercados y clases de empresas. Para Revilla y Fernández (2011) los instrumentos de evaluación de la RSE deben ser coherentes con los estándares de medición que requieren para su respectiva evaluación dentro del contexto en que se desarrolla la empresa.

\section{Sector de la construcción en Bucaramanga}

Las mipymes (medianas y pequeñas empresas) del sector de la construcción en Bucaramanga han presentado un proceso de desaceleración económica según el Departamento Administrativo Nacional de Estadística [DANE] 2016, donde el área de licenciamiento para la construcción bajó en 40,0\% en abril del 2016, cuando se licenciaron $1961272 \mathrm{~m}^{2}$, mientras que igual periodo de 2015 fue de $2757880 \mathrm{~m}^{2}$. En cuanto al área aprobada para vivienda diferente de interés social en abril de 2016 fue de $1468988 \mathrm{~m}^{2}, \mathrm{y}$, para vivienda de interés social el área aprobada fue de $305010 \mathrm{~m}^{2}$, mientras que en abril de 2015 fue de $562004 \mathrm{~m}^{2}$. Sin embargo, este panorama se mejora con el incremento del producto interno bruto (PIB), que para el primer trimestre del 2016 mostró un crecimiento del 2,5\% en comparación con el mismo trimestre del 2015. Además, la generación de empleo en Colombia de forma directa e indirecta en el sector de la construcción empleó en abril de 2016 a 1428000 personas, con un aumento de $10,3 \%$ frente al mismo mes de 2014. (DANE, 2016) 


\section{Método}

\section{Tipo de estudio}

La investigación tiene un alcance descriptivoy un enfoque cualitativo, donde se hace la observación del objeto de estudio mediante el análisis de sus características, su estructura organizacional, y el movimiento de las empresas del sector de la construcción, con la finalidad de conocer y medir la información recogida para formular conclusiones relevantes. (Fernández, 2009)

\section{Participantes}

La población del sector de la construcción está representada por 260 empresas registradas en la Cámara de Comercio de Bucaramanga en el año 2013, de donde se seleccionaron 60 para realizar el estudio a través de una muestra determinada por juicio y conveniencia (Mendoza, 2013), considerando los siguientes factores: ubicación geográfica, nivel de productividad, antigüedad en el sector e inscripciones-renovaciones en la Cámara de Comercio de Bucaramanga al año 2013. El total de empresas a analizar se escogió por medio de bases estadísticas que permitieron establecer la población ideal para la toma de un registro de información y la aplicación del instrumento para la recolección de la información. (Bencardino, 2012)

\section{Materiales e instrumentos}

El instrumento de recolección de la información consta de ochenta preguntas distribuidas en nueve bloques que representan las variables de medición: 1. Organización y gestión de empresas; 2. Promoción, comunicación y trasparencia; 3. Gestión normas de procesos y acreditación; 4. Autoevaluación / Desempeño; 5. Grupo de interés interno; 6. Grupo de interés externo (entorno); 7. Grupo de interés externo (contexto); 8. Modelo de negocio actual; y, 9. Sistema de contratación. La recolección de los datos se hizo a través de entrevistas direccionadas al nivel estratégico de las empresas. Las preguntas se establecieron bajo la escala de Likert de 1 a 4 ( 1 = nada; ...; 4 = bastante). Las técnicas estadísticas utilizadas fueron descriptivas para la contextualización del sector y correlaciones bivariadas con el estadístico Tau-b de Kendall para el tratamiento de variables nominales. Los análisis se realizaron con el paquete estadístico Statgraphics.

\section{Resultados}

Los empresarios, frente a la responsabilidad social empresarial en el sector de la construcción en la ciudad de Bucaramanga, evidencian un nivel de satisfacción en el nivel bastante destacándose la percepción del cliente interno del sector de la construcción sobre la implementación de la RSE (ver Figura 1) con respecto a los tres stakeholders-internos: socios y aliados (54\%), trabajadores (50\%) y accionistas (44\%). Es evidente que el hecho de que solo un poco más de la mitad de los clientes-internos estén de acuerdo con la forma en que se implementa la RSE implica la necesidad de mejorar estos procesos para el desarrollo de la competitividad en el sector.

En cuanto al nivel Mucho, que indica una aceptación de la satisfacción en un nivel medio de aprobación, los resultados muestran una deficiencia en cada grupo de los stakeholders, en tanto que el valor porcentual de mayor impacto lo asumen los trabajadores y accionistas, (28\%), porcentaje precario para el cumplimiento de la sostenibilidad del sector. De otra parte, se destaca una aceptación de la satisfacción en un nivel medio de aprobación por la deficiencia en cada grupo de los stakeholders, donde el $28 \%$ de los trabajadores y accionistas señalan como precario el cumplimiento de la sostenibilidad del sector; por otro lado, el $26 \%$ de los socios indican que están insatisfechos con el alcance de la RSE en todo el sector de la construcción en la ciudad de Bucaramanga y su área metropolitana, implicando este aspecto la necesidad de establecer políticas que favorezcan su adopción.

Es importante indicar que en el nivel poco y en el nivel Nada quienes no están satisfechos son los accionistas (20\% y $8 \%$, respectivamente).

De otro lado, con respecto a sus stakeholders-externos, se detectó que los empresarios consideran que existen falencias en las responsabilidades compartidas, como se evidencia en el nivel Nada con las ONG (62\%) y con los sindicatos (58\%). Mientras que en el nivel Bastante los empresarios indican que comparten responsabilidades sobre todo con los clientes (48\%), la empresa (48\%), el gobierno (46\%), los proveedores (42\%), los distribuidores (38\%), los acreedores (46\%), los competidores (34\%), los contratistas (32\%) y la comunidad (30\%); y en el nivel mucho con los contratistas (46\%), los clientes (34\%), los proveedores (32\%), el gobierno (32\%) y la empresa (30\%), como se evidencia en la Tabla 1.

Es evidente que la empresa dirige sus esfuerzos hacia el cliente externo, porque es en ese camino que encuentra opciones de mejora frente a la competencia; sin embargo, está descuidando su cliente interno. 


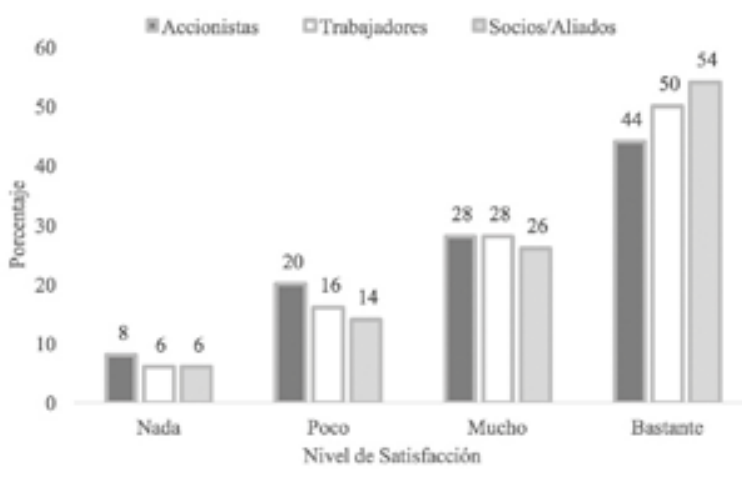

Figura 1.

Distribución del nivel de importancia que otorgan los empresarios al cliente interno en el sector de la construcción

Tabla 1

Distribución de la percepción sobre las falencias en las responsabilidades compartidas según los empresarios del sector de la construcción

\begin{tabular}{ccccc}
\hline Stakeholders & Nada & Poco & Mucho & Bastante \\
\hline Clientes & 8 & 10 & 34 & 48 \\
Proveedores & 10 & 16 & 32 & 42 \\
Distribuidores & 20 & 14 & 28 & 38 \\
Contratistas & 4 & 18 & 46 & 32 \\
Acreedores & 16 & 24 & 24 & 36 \\
Competidores & 18 & 22 & 26 & 34 \\
Sindicatos & 58 & 16 & 16 & 10 \\
Comunidad & 10 & 34 & 26 & 30 \\
Gobierno & 2 & 20 & 32 & 46 \\
Empresa & 10 & 12 & 30 & 48 \\
ONG & 62 & 4 & 14 & 20 \\
\hline
\end{tabular}

Fuente: Los Autores

\section{Sobre los stakeholders internos}

Con la utilización de las correlaciones bivariadas y el estadístico Tau-b de Kendall se detectó que hay una correlación positiva y significativa al $99.0 \%$ en las variables La empresa satisface necesidades de manera socialmente responsable con sus accionistas y La empresa satisface necesidades de manera socialmente responsable con sus trabajadores ( $\mathrm{W}=0.750 ; \mathrm{p}<0.000)$, y con $L a$ empresa satisface necesidades de manera socialmente responsable con sus socios/aliados ( $\mathrm{W}=0.755 ; \mathrm{p}<0.000)$, y entre esta última y La empresa satisface necesidades de manera socialmente responsable con sus trabajadores $(\mathrm{W}=0.928 ; \mathrm{p}<0.000)$. Las anteriores relaciones indican que los stakeholders-internos se relacionan entre sí porque ven un interés mutuo. Esto evidencia que la empresa dirige sus esfuerzos hacia sus trabajadores, pero al revisar más profundamente las respuestas entregadas por los empresarios entrevistados se observa que subvaloran el talento que poseen en su interior y pasan a valorar solo lo relacionado con el clienteexterno, descuidando sus stakeholders-internos, quienes en últimas son los encargados de diseminar la imagen de la compañía frente a los diferentes mercados y clientes externos de la empresa.

\section{Comentarios}

Los grupos de interés, internos y externos, muestran relación en la satisfacción de sus necesidades como una organización socialmente responsable, en niveles similares, en los stakeholders, donde la relación es más continua y favorable para el desarrollo de sus actividades.

Para los empresarios del sector de la construcción, la importancia que le otorgan a los procesos organizacionales se centra en los accionistas y en los socios de la compañía, descuidando a los trabajadores, con quienes deben compartirla, considerando que son miembros activos en todo el proceso de la cadena productiva y que son la base fundamental para el desarrollo de la responsabilidad social empresarial, que emerge desde el centro empresarial para manifestarse positivamente en el contexto exterior de la compañía.

Se observa que el sector de la construcción en Bucaramanga tiene una relación con otros stakeholders externos, donde se presentan niveles no compartidos y comprometidos en diferentes esferas, como es el caso de las relaciones con las organizaciones no gubernamentales y los sindicatos, entre otras. Esta situación genera falta de interés por las responsabilidades adquiridas y compartidas para el desarrollo y cumplimiento de una RSE efectiva en todo el proceso que se desarrolla en las empresas del sector de la construcción.

El cumplimiento responsable de las empresas del sector de la construcción se identifica en el desarrollo relacionado con los grupos de interés internos y externos, evidencia de un sector sólido y comprometido en toda la RSE, que permite aminorar la brecha del desempleo de la región, aportando valores positivos en toda la cadena de producción, de la comercialización y de la contratación, en línea con el desarrollo regional, en empresas socialmente responsables.

Frente a este panorama se recomienda a las empresas del sector de la construcción en Bucaramanga seguir las siguientes buenas prácticas: considerar a sus stakeholders internos y a la RSE como una dupla para alcanzar la competitividad, y de esta manera fomentar 
el desarrollo en la formación para los grupos de interés interno y externo, para consolidar los procesos y las actividades de la empresa, generando valor agregado en cada uno de sus métodos y convirtiéndose en empresas competitivas. Además, las organizaciones han de velar por la intensificación de los procesos de investigación, desarrollo e innovación, I+D+i, en áreas que permitan seguir avanzando en el conocimiento y en el perfeccionamiento de la cadena productiva dentro del marco de la RSE para potencializar los recursos de la región.

Otro aspecto importante que se debe considerar en las organizaciones que son socialmente responsables con su cliente externo, es la generación de espacios de comunicación para que los stakeholders-internos se apropien de los procesos y de las políticas organizacionales de la empresa, buscando alcanzar un mayor conocimiento entre los grupos involucrados. En este sentido, las empresas pueden ver la disminución de las brechas que impiden el desarrollo empresarial y buscar el favorecimiento de la creación de espacios de comunicación a partir del trabajo en equipo, fortaleciendo el sector de la construcción como oferente de empresas que son modelos para el cumplimiento y el desarrollo de la RSE.

Es necesario que las empresas de Santander propendan por el desarrollo de espacios que faciliten la integración de los stakeholders-internos en los procesos empresariales, favoreciendo su llegada oportuna hacia el exterior y aportando al mejoramiento de la imagen de la empresa. Todo esto considerando a los stakeholders-internos como implicados en la apropiación de las políticas de la organización.

Para finalizar, es importante indicar que las empresas del sector de la construcción, asícomo las diferentes empresas de la región deben concientizarse de la importancia que tienen los stakeholders internos y externos, pues son actores primordiales en el cumplimiento de los objetivos organizacionales y de la mejora de la imagen corporativa de la organización en el entorno.

\section{Referencias}

Aguilera, A. \& Puerto, P. (2012). Crecimiento empresarial basado en la Responsabilidad Social. Pensamiento \& Gestión, (32), 1-26.

Accinelli, E. \& de la Fuente, J. (2013). Responsabilidad social corporativa, actividades empresariales y desarrollo sustentable: Modelo matemático de las decisiones en la empresa. Contaduría y Administración, 58(3), 227-248.
Bateman, S. \& Snell, A. (2005). Administración: Un nuevo panorama competitivo (4a. ed., pp.147, 150-151). México: McGraw-Hill/Interamericana.

Bestratén, M. \& Pujol, L. (2004). Responsabilidad social de las empresas (I y II). Notas Técnicas de Prevención-NTP, (643).

Caamaño, A. (2012). Responsabilidad social empresarial e implicaciones para el trabajo social. Recuperado de http:// gredos.usal.es/jspui/bitstream/10366/122130/3/TG_ Caama\%C3\%B1oRueda_Responsabilidad.pdf

Correa, E., Flynn, S. \& Amit, A. (2004). Responsabilidad social corporativa en América Latina: una visión empresarial. United Nations Publications.

Departamento Administrativo Nacional de Estadística (2016). Proyecto de investigación del sector de la construcción de edificaciones en Colombia. Recuperado de http://www. dane.gov.co/files/investigaciones/boletines/pib_const/ Bol_ieac_Itrim16.pdf

ETHOS. (2011). Indicadores de Responsabilidad Social Empresarial. Recuperado de http://www1.ethos.org.br/ EthosWeb/arquivo/0-Abbe2011_Indic_ETHOS_ESP.pdf

Fernández, P. (2009). La Investigación Cualitativa. Sistema.

García, T., Llarena, R., Chaín, P., Contreras, S., Méndez, S., Herrerías, A., \& Reyna, L. (2016). Implementación de iniciativas de Responsabilidad Social en las pequeñas y medianas empresas: construcción de un instrumento de medición de RSE en Las Pymes mexicanas. Revista Global de Negocios, 4(5), 21-38. Recuperado de http://www. theibfr2.com/RePEc/ibf/rgnego/rgn-v4n6-2016/RGNV4N6-2016-2.pdf

Guédez, V. (2008). Ser confiable responsabilidad social y reputación empresarial. Planeta.

Jiménez, R., Alcaraz, A. \& Leco, C. (noviembre, 2015). La educación como factor de desarrollo social, de los stakeholders obreros de la empresa manufacturera de calzado en el municipio de León, Guanajuato. 20 Encuentro Nacional sobre Desarrollo Regional en México. AmeciderCRIM, UNAM: Cuernavaca.

Kowszyk, Y., Covarrubias, A. \& García, L. (2011). El estado de la responsabilidad social empresarial en América Latina 2011: Percepciones de los consumidores y ejecutivos de empresas. In Red Forum Empresa.

Bencardino, M. (2012). Estadística y Muestreo. Bogotá: ECOE Ediciones Ltda. 
Mendoza, J. (2013). Uso de las tecnologías de la información y comunicación en los procesos de la cadena de valor en las empresas del sector cacaotero. Revista Economía y Administración, 4, 17-29.

Meza, A. (2011). Indicadores sobre responsabilidad Social Corporativa. Recuperado de direccionestrategica.itam.mx/ wpcontent/.../06/IndicadoresRSCOK.doc

McWilliams, A., Siegel, S. \& Wright, M. (2006). Corporate social responsibility: International perspectives. Recuperado de http://papers.ssrn.com/sol3/papers.cfm?abstract_ $\mathrm{id}=900834$

Navarro, F. (2012). Responsabilidad social corporativa. Madrid: ESIC Editorial.

Perlines, H. (2015). El efecto mediador de la RSE en la relación de la orientación emprendedora y el desempeño de las cooperativas agroalimentarias. CIRIEC, (85), 1-29. Recuperado de https://www. researchgate.net/profile/Felipe_Hernandez- Perlines/ publication/288511799_El_efecto_mediador_de_la_RSE_ en_la_relacion_de_la_orientacion_emprendedora_y_ el_desempeno_de_las_cooperativas_agroalimentarias/ links/56c0c6a308aeedba05647dc6.pdf

Revilla, G. \& Fernández, R. (2011). La gestión de los grupos de interés (stakeholders) en la estrategia de las organizaciones. Economía Industrial, (381), 71-76.
Ríos, G. (2013). Responsabilidad social y gestión del conocimiento como estrategias de gestión humana. Estudios Gerenciales, 29(126), 110-117.

Solano, D. (2005). Responsabilidad Social Corporativa: qué se hace y qué debe hacerse. Cuadernos de Difusión, 10(1819), 163-165.

Silos, J. \& Galiano, P. (2011). Informe Forética 2011. Evolución de la responsabilidad social de las empresas en España. Madrid: Forética.

Suárez, P. (2011). Responsabilidad social empresarial del Ecuador casos de aplicación. (Tesis de pregrado). Universidad de Cuenca, Cuenca, Ecuador.

Ulloque, I. \& Berrío, M. (2014). Diseño de un programa de responsabilidad social empresarial dirigido al público interno de la Agencia de Aduanas Gama SA Nivel 1. (Disertación Doctoral), Universidad de Cartagena.

Unión Europea. (2001). Libro verde: fomentar un marco europeo para la responsabilidad social de las empresas. Bruselas: Comisión de las Comunidades Europeas. Recuperado de www.europa.eu.int/comm/off/green/index-es.htm

Yfarraguirre, L. (2014). Necesidad de Indicadores de Medición de la Responsabilidad Social en las Universidades. Daena: International Journal of Good Conscience, 91(1), 142-157. 